\title{
Human papillomavirus infection and cervical dysplasia in female sex workers in Northeast China: an observational study
}

\author{
Haiqing Jia*, Xiaobin Wang, Zaiqiu Long and Liankun Li
}

\begin{abstract}
Background: Women having multiple sex partners are reportedly at an increased risk of HPV infection. However, the prevalence and risk factors of HPV infection in female sex workers (FSWs) vary considerably across racial/ethnic, socioeconomic, and geographic groups. This study aimed to determine the prevalence and risk factors of HPV infection in FSWs in Northeast China.

Methods: A total of 309 FSWs identified and approached through a local police office and 1000 healthy subjects from a single factor undergoing annual gynecological examinations in Shenyang were recruited. A liquid-based ThinPrep Pap test and the Hybrid Capture II-based high-risk HPV DNA test, with or without a colposcopic examination, were performed on both FSWs and control subjects. Data on HPV infection and histological and cytological lesions of the cervix were obtained and analyzed. A questionnaire survey was administered to all 309 FSWs with their sociodemographic and behavioral information collected. The association of various socio-demographic and behavioral variables with HPV infection was assessed.
\end{abstract}

Results: HPV was significantly more prevalent in FSWs (61.90\%) than in healthy control subjects $(21.00 \%)(P<0.01)$, so were cervical lesions $(P<0.01)$. HPV prevalence in our sample of FSWs fell in the upper range of reported values in FSWs across different countries, and was similar to that for FSWs in the southeast Chinese city of Huzhou but higher than that for FSWs in southwest China, Guangxi, as compared with data from other studies within China. HPV infection in FSWs was significantly associated with the age at first sexual intercourse (OR 0.699, $95 \% \mathrm{Cl} 0.492-0.992)$ and postmenopause (OR 2.928, 95 \% Cl 1.099-7.800) $(P<0.05)$.

Conclusions: FSWs are at a substantially high risk of HPV infection and cervical dysplasia development as compared with healthy control subjects in Shenyang, China. Age of first sexual intercourse and post-menopause are two independent risk factors for HPV infection in this special group of population. Intensified and coordinated efforts from government, public health sector, communities and families are needed to reduce the risk of HPV infection in this specific group of population.

Keywords: Female sex workers, Human papillomavirus (HPV), Cervical dysplasia, Risk factors, Shenyang, China

\footnotetext{
*Correspondence: haiqing725@163.com

Department of Gynecological Oncology, Liaoning Provincial Cancer Hospital,

44 Xiaoheyan Road, Shenyang, Liaoning 110042, China
} 


\section{Background}

Cervical cancer is one of the most common causes of cancer death in women, claiming more than 270,000 lives annually worldwide [1]. One of the most common types of cervical cancer is squamous cell carcinoma. Cervical dysplasia or cervical intraepithelial neoplasia (CIN) is the precursor of invasive squamous carcinoma of the cervix. Histologically, CIN is graded into mild dysplasia (CIN 1), moderate dysplasia (CIN 2), or severe dysplasia (CIN 3). Cytologically, cervical dysplasia is further categorized with the Bethesda system as: Atypical squamous cells of undetermined significance (ASC-US), atypical squamous cells - cannot exclude high-grade squamous intra-epithelial lesion HSIL (ASC-H), low grade squamous intraepithelial lesion (LGSIL or LSIL) consisting atypia and CIN 1, high grade squamous intraepithelial lesion (HGSIL or HSIL) primarily consisting of CIN 2-3 plus carcinoma, squamous cell carcinoma, atypical glandular cells not otherwise specified (AGC-NOS), atypical glandular cells suspicious for adenocarcinoma in situ (AIS) or cancer (AGC-neoplastic), and AIS. atypical squamous cells of undetermined significance (ASC-US), atypical squamous sells, Cannot Rule Out High-Grade Squamous Intra-epithelial Lesion (ASC-H) [2].

Genital infection with human papillomavirus (HPV), particularly the high-risk subtypes HPV-16 and HPV-18, is playing a major role in the etiopathogenesis of cervical cancer [3]; although the virus alone may not be sufficient to cause cancer [4], virtually all cervical cancers are associated with persistent infection with one of the highrisk types of HPV.

Since HPV is predominantly transmitted through sexual contact, HPV infection in women varies greatly with sexual activities and the number of sexual partners $[5,6]$. In a recent comprehensive review paper, Soohoo et al. summarized data on the difference in HPV prevalence between female sex workers (FSWs) and the general population and on variations in HPV prevalence across different geographical regions of the world and showed that the median overall prevalence of HPV infection in FSWs in six WHO defined regions (i.e., Africa, Americas, Eastern Mediterranean, Europe, Southeast Asia and Western Pacific) was $42.7 \%$, significantly higher than that in the general population of women [7]. Moreover, these authors showed huge geographic variations (ranging from 2.3 to $100 \%$ ) in HPV prevalence in FSWs among the regions assessed. Nevertheless, of the 35 studies included in this review paper only one was from China, involving 810 female sex workers from Guangxi, a province in southwest China [8]. To date, no reports are available on the HPV infection and cervical dysplasia in FSWs in Shenyang, Liaoning, a province in Northeast China. Moreover, risk factors for HPV infection in FSWs in Shenyang have not been well defined, although the association of HPV infection with various demographic and behavioral variables has been assessed in FSWs in other studies [9-11].

This study was therefore conducted with two objectives: 1) to assess the prevalence of HPV infection and incidence of CIN in FSWs in Shenyang, China; and 2) to define putative risk factors for HPV infection in this unique group of population in Shenyang.

\section{Methods}

\section{Ethical statement}

The study was reviewed and approved by the Institutional Review Board of Liaoning Provincial Cancer Hospital, Shenyang, China and conducted in compliance with the ethical principles for medical research involving human subjects stated in the World Medical Association Declaration of Helsinki. Informed consent was obtained from all subjects.

\section{Subjects}

A total of 310 FSWs who were detained between October and December, 2013 for alleged engagement in illegal prostitution or sexual acts in public entertainment and leisure facilities (e.g., bars, karaoke rooms, night clubs, beauty spas, salons, saunas, foot and massage parlors) by police in Shenyang, China were identified and approached with the help from of a local police branch. They were all mentally healthy and non-pregnant without structural and anatomic malformations in the reproductive tract and without a history of pelvic irradiation. They had not been screened for cervical neoplasia within five years prior to their police detainment. After exclusion of one subject who refused to undergo gynecological examination, 309 FSWs were finally recruited to the study. As a representative sample of the general population, 1000 women who underwent free annual gynecologic examinations in December, 2013 in the Department of Gynecological Oncology, Liaoning Provincial Cancer Hospital were included for the purpose of normal control. They were employees from a single factory in Shenyany. No specific inclusion and exclusion criteria were applied to the selection of control subjects as a random sample of the general population.

There was no difference in the cultural and racial/ethnic backgrounds between FSWs and control subjects; they were all Han Chinese.

\section{Papanicolaou (Pap) test and HPV testing}

A liquid-based ThinPrep Pap test was performed as previously described [12] and high-risk oncogenic HPV was assessed by the Digene Hybrid Capture II assay (Digene Corporation, Gaithersburg, MD, USA) using a mixture of probes including $16,18,31,33,35,39,45,51,52,56$, 58,59 and 68 as instructed by the manufacturer on the 309 FSWs at the time of their police detainment and on 
the 1000 healthy control subjects at the time of their regular gynecologic/obstetric visits. Cytological abnormalities revealed by Pap test were classified according to the Bethesda System. When ASCUS or higher grades of squamous intraepithelial lesion were detected, regardless of the HPV testing results (positive or negative), or when HPV was positive, regardless of cytological abnormalities, immediate colposcopy was conducted along with visual inspection with acetic acid (VIA) or Lugol's iodine (VILI). When colposcopy showed abnormalities, biopsies were taken from transformation zones and acetowhite areas and sent to the Department of Pathology of Liaoning Tumor Hospital for histopathologic assessment. Suspected CIN was graded as mild, moderate or severe based on cytologic findings in the Pap test and/or histologic findings from the colposcopic examination.

\section{Interview survey}

A standard risk factor assessment interview was conducted on 309 FSWs in person at the time of their police detainment. Data on their basic demographics, sexual activities and behaviors (including the age of the first sexual intercourse), reproductive history, menarche, sexually transmitted disease (STD) history, and smoking habit (past and present) were collected.

\section{Statistical analysis}

Data on the prevalence rates of HPV and CIN in the two groups of subjects were analyzed by Pearson's chi-square test. Putative risk factors for HPV infection were analyzed by univariate and multivariate logistic regression analyses. Differences were considered significant when $P<0.05$. The statistical software SPSS 11.5 (Chicago, IL, USA) was used.

\section{Results}

HPV infection and intraepithelial lesions in control subjects and FSWs

Presented in Table 1 are data on HPV infection, cytological abnormalities and cervical intraepithelial cell

Table 1 Differences in HPV infection and cervical intraepithelial lesions between healthy control subjects and FSWs analyzed by Pearson's chi-square test

\begin{tabular}{lcc}
\hline & Controls $(n=1000)$ & Sex workers $(n=309)$ \\
\hline HPV infection & $21.00 \%$ & $61.90 \% \%^{* *}$ \\
ASCUS & $2.70 \%$ & $32.04 \%^{* *}$ \\
ASCUS-H & $0.20 \%$ & $4.21 \%^{* *}$ \\
CIN1 & $2.90 \%$ & $8.41 \%^{*}$ \\
CIN2 & $1.00 \%$ & $5.50 \% \%^{* *}$ \\
CIN3 & $0.50 \%$ & $2.91 \%{ }^{* *}$ \\
\hline
\end{tabular}

Single $\left(^{*}\right)$ and double ${ }^{* *}$ ) asterisks indicate significant differences from control subjects at $P<0.05$ and at $P<0.01$ respectively lesions in FSWS and healthy control subjects assessed. The rate of HPV infection was significantly higher in FSWs than in healthy control subjects $(P<0.01)$. Cytological abnormalities, both ASCUS and ASCUS-H, were detected in a significantly higher proportion in FSWs than in control subjects $(P<0.01)$. In consistence with HPV infection and abnormal cytology, significantly higher occurrence of CIN $1(P<0.05)$, CIN $2(P<0.01)$ or CIN $3(P<0.01)$ was observed in FSWs than in control subjects.

\section{HPV infection in different age groups of female sex workers}

To assess the possible age dependency of HPV infection in FSWs, we stratified the 309 female sex workers into 5 different age groups. As shown in Table 2, the prevalence rate of HPV infection ranged from 40.07 to $85.71 \%$; FSWs either younger than 21 or older than 51 years were at significantly higher risk of HPV infection.

\section{Risk factors for HPV infection in FSWs}

To identify putative risk factors for HPV infection in FSWs, we first performed univariate regression analysis and the results are presented in Table 3. HPV infection in FSWs was found not significantly associated with marital status, education, smoking, abortion, previous sexual disease, condom use, contraception pills, age of first period, time participating in commercial sex work, vaginal medication use, and regular obstetrical and gynecological examinations. In contrast, young age of first sexual intercourse was the only variable that significantly influenced HPV infection $(P<0.001)$.

We next performed multivariate logistic regression analysis. The results clearly revealed that HPV infection in FSWs was significantly associated with the age of first sexual intercourse and post-menopause while the other variables assessed had no effect (Table 4).

\section{Discussion}

Routine HPV screening for women aged 21 and 65 years can reduce the chance of cervical cancer development [13]. Various HPV testing modalities are available [14]. In this study, we chose the Digene Hybrid Capture 2

Table 2 Age stratification of HPV infection in FSWs

\begin{tabular}{lcc}
\hline Age (years) & $\mathrm{N}$ & HPV prevalence \\
\hline$\leq 20$ & 21 & $61.90 \% *$ \\
$21-30$ & 81 & $41.98 \%$ \\
$31-40$ & 112 & $41.07 \%$ \\
$41-50$ & 88 & $52.27 \%$ \\
$>51$ & 7 & $85.71 \% *$ \\
\hline
\end{tabular}

Single $(*)$ asterisk indicates a significant difference from control subjects at $P<0.05$ 
Table 3 Results of univariate logistic regression analysis of risk factors for HPV infection in FSWs

\begin{tabular}{|c|c|c|c|c|}
\hline Category & $\mathrm{HPV}(+)$ & HPV (-) & $\begin{array}{l}\text { Odds ratio } \\
(95 \% \mathrm{Cl})\end{array}$ & $P$ \\
\hline \multicolumn{5}{|l|}{ Marital status } \\
\hline Single & 29 & 28 & 1 & \\
\hline Married & 55 & 63 & $0.84(0.45-1.59)$ & 0.39 \\
\hline Widowed & 7 & 4 & $1.69(0.45-6.41)$ & 0.49 \\
\hline Divorced & 54 & 69 & $0.76(0.40-1.42)$ & 0.10 \\
\hline \multicolumn{5}{|l|}{ Education } \\
\hline$\leq$ Middle school & 110 & 132 & 1 & \\
\hline$\geq$ High school & 35 & 32 & $1.31(0.76-2.26)$ & 0.10 \\
\hline \multicolumn{5}{|l|}{ Active smoking } \\
\hline No & 100 & 110 & 1 & \\
\hline Yes & 45 & 54 & $0.92(0.57-1.48)$ & 0.76 \\
\hline \multicolumn{5}{|c|}{ Second hand smoking } \\
\hline No & 76 & 76 & 1 & \\
\hline Yes & 69 & 88 & $0.78(0.50-1.23)$ & 0.25 \\
\hline
\end{tabular}

Number of abortion

$\begin{array}{lrrll}\leq 3 & 130 & 136 & 1 & \\ >3 & 15 & 28 & 0.56(0.29-1.10) & 0.11 \\ \text { Sexual disease history } & & & & \end{array}$

Sexual disease history

$$
\text { No }
$$

Condom use

$$
\begin{aligned}
& \text { No } \\
& \text { Yes }
\end{aligned}
$$

Contraception pills

No

Yes

Age of first period (years)

$$
\begin{aligned}
& \leq 13 \\
& 14-15 \\
& 16-17
\end{aligned}
$$$$
\geq 18
$$

Age of 1st sexual intercourse

$$
\begin{aligned}
& \leq 20 \text { years } \\
& 21-23 \text { years } \\
& \geq 24 \text { years }
\end{aligned}
$$

Time as a sex worker

$$
\begin{aligned}
& <1 \text { year } \\
& \geq 1 \text { year }
\end{aligned}
$$

Use of vaginal medications

$$
\text { No }
$$$$
\text { Yes }
$$

$$
\begin{array}{rrll}
137 & 148 & 1 & \\
8 & 16 & 0.54(0.22-1.30) & 0.19
\end{array}
$$

\begin{tabular}{|c|c|c|}
\hline 92 & 102 & 1 \\
\hline 53 & 62 & $0.95(0.60-1.50)$ \\
\hline
\end{tabular}

$\begin{array}{rrll}29 & 34 & 1 \\ 116 & 130 & 1.05(0.60-1.82) & 0.73\end{array}$

$\begin{array}{llll}116 & 130 & 1.05(0.60-1.82) & 0.73\end{array}$

$\begin{array}{rrll}109 & 121 & 1 \\ 36 & 43 & 0.93(0.56-1.55) & 0.84\end{array}$

$36-43-0.93(0.56-1.55) \quad 0.84$

$\begin{array}{llll}28 & 32 & 1 & 0.97\end{array}$

$\begin{array}{llll}58 & 69 & 0.96(0.52-1.78) & 0.96\end{array}$

$43 \quad 43 \quad 1.14(0.59-2.21) \quad 0.77$

$16 \quad 20 \quad 0.91(0.40-2.10) \quad 0.73$

$\begin{array}{llll}85 & 72 & 1 & 0.00\end{array}$

$47 \quad 80 \quad 0.40(0.24-0.67) \quad 0.12$

$\begin{array}{llll}13 & 12 & 0.99(0.53-1.85) & 0.81\end{array}$

$114 \quad 127 \quad 1$

$31 \quad 37 \quad 0.93(0.54-1.60) \quad 0.93$

$\begin{array}{llll}31 & 0.93 & -9.54-1.60) & 0.93\end{array}$

$85+72-1000$

Table 3 Results of univariate logistic regression analysis of risk factors for HPV infection in FSWs (Continued)

\begin{tabular}{lrrll}
\hline Regular Ob/Gyn exams & & & & \\
No & 95 & 115 & 1 & \\
Yes & 50 & 49 & $1.24(0.77-1.99)$ & 0.46 \\
Menopause & & & & \\
No & 131 & 156 & 1 & \\
Yes & 14 & 8 & $0.85-5.12$ & 0.11 \\
\hline
\end{tabular}

High-Risk HPV DNA Test, an approach approved by the US Food and Drug Administration for use in cervical cancer screening, that is highly specific, reproducible, sensitive and reliable [15-17]. With this test, we detected high-risk HPV DNA in $61.90 \%$ of FSWs and in $21.00 \%$ of healthy control subjects (Table 1). It has been documented that there are huge variations in HPV infection in FSWs between different geographic areas and different races. While over $80 \%$ of FSWs were HPVpositive in southern Vietnam [18] and Hungary [19], HPV DNA was detected in $66.8 \%$ of FSWs $(n=200)$ in Peru [20], 57.2 \% of FSWs $(n=369)$ in Philippines [21], $49.5 \%$ of FSWs $(n=281)$ in northern Vietnam [22], $47 \%$ of FSWs $(N=148)$ in South Korea [23], $31.6 \%$ of FSWs $(n=288)$ in Australia [24], $22.9 \%$ of FSWs $(n=254)$ in Thailand [25] and $14.4 \%$ of FSWs $(n=187)$ in Singapore [26]. Compared with these worldwide data, the prevalence rate of HPV infection in FSWs detected in this study was in the upper range. Compared with reports from other Chinese studies, the HPV infection rate in FSWs in Shenyang was very close to that in FSWs in Huzhou (66.7 \%) [11], but was much higher than that in FSWs in Guangxi (38.9 \%) [8]. Currently, the exact reasons underlying the reported difference in HPV prevalence

Table 4 Results of multivariate logistic regression analysis of risk factors HPV infection in FSWs

\begin{tabular}{lcccc}
\hline Variables & $\begin{array}{l}\text { Regression } \\
\text { coefficient }\end{array}$ & $\begin{array}{l}\text { Odds } \\
\text { ratio }\end{array}$ & $95 \% \mathrm{Cl}$ & $P$ value \\
\hline Marital status & -0.067 & 0.935 & $0.727-1.024$ & 0.604 \\
Education & 0.445 & 1.561 & $0.869-2.805$ & 0.136 \\
Sexual disease history & -0.689 & 0.502 & $0.194-1.301$ & 0.156 \\
Smoking & -0.087 & 0.917 & $0.550-1.528$ & 0.738 \\
Second hand smoking & -0.279 & 0.756 & $0.466-1.227$ & 0.258 \\
Age of first period & -0.004 & 0.996 & $0.762-1.301$ & 0.976 \\
Post-Menopause & 1.074 & 2.928 & $1.099-7.800$ & 0.032 \\
Number of abortion & -0.601 & 0.548 & $0.267-1.127$ & 0.102 \\
Condon use & 0.093 & 1.097 & $0.598-2.012$ & 0.764 \\
Contraception pills & -0.120 & 0.887 & $0.497-1.583$ & 0.685 \\
Age of first sex & -0.359 & 0.699 & $0.492-0.992$ & 0.045 \\
Time as a sex worker & 0.089 & 1.093 & $0.600-1.991$ & 0.772 \\
\hline
\end{tabular}


in FSWs in different areas in China are unknown and further studies are warranted.

In consistency with HPV infection, cervical dysplasia, assessed either histologically or cytologically, was significantly more prevalent in FSWs than in healthy controls in this study (Table 1). In the current literature, data on cervical epithelial cell lesions in FSWs are scarce. In a study involving $90 \mathrm{FSWs}$ aged 18 to 58 years in Antananarivo, Madagascar, the prevalence was $3.3 \%$ for low-grade squamous intraepithelial lesions and $18.9 \%$ for ASCUS while no high-grade lesion was detected [27]. In another study from Hong Kang, CIN 1-3 lesions were observed in $9.8 \%$ of 235 FSWs [28]. Comparatively, squamous intraepithelial lesions, particularly ASC-US (32.04\%) and low-grade lesions, detected in this study were all higher than those reported in the two studies mentioned above. It has been well documented that free-of-charge screening services to FSWs is very helpful not only in early detection and proper follow-up in case of abnormal Pap tests but also in increasing the awareness of women's health issues [29]. Given the substantially high incidence of ASC-US and low-grade lesions observed in this study, a free or practically feasible and cost-efficient HPV screening program is urgently needed to minimize the risk for cervical cancer in FSWs in Shenyang.

Numerous factors have been assessed for a putative link with HPV infection in FSWs in previous studies. However, inconsistent results have been obtained. In this study, we assessed various socio-demographic and behavioral variables, among which age of first sexual intercourse and menopause were the only two risk factors for HPV infection in FSWs in Shenyang.

In agreement with our findings, young age (OR 0.699, $95 \%$ CI 0.492-0.992) and menopause (OR 2.928, 95 \% CI 1.099-7.800) have been suggested as independent risk factors for HPV infection in FSWs in previous studies $[8,10,22,24,30]$. The exact mechanisms underlying increased vulnerability to HPV infection in young and post-menopausal FSWs are not fully understood. However, inadequate acquired immunity at young age and decreased acquired immunity at post-menopausal age might be one of the explanations [30]. Supporting this speculation, Sivro and colleagues have demonstrated that there are significant hormone-associated changes in systemic and mucosal cytokine/chemokine production, which may have implications for the age-related decline in the ability to fight against infections in young and post-menopausal women [31].

Education levels $[10,18]$ and years of commercial sex work [10] have been demonstrated to affect HPV infection in FSWs in other studies. Inconsistent with these studies, the present study showed that HPV infection in FSWs in Shenyang was not associated with education levels or years of commercial sex work.
The impact of cigarette smoking on HPV prevalence has been assessed in both men and women [32]. However, in the current literature, little information is available regarding the difference in HPV infection between smoking and non-smoking FSWs, although it has been reported that the risk for FSWs to develop preinvasive or invasive cervical lesions increase with the number of cigarettes they smoked per day and their years of smoking [33]. In this study, the effect of smoking, either active or passive, on HPV infection was not demonstrated in FSWs in Shenyang and warrants further assessment in the future.

The benefit of condom use in protecting HPV infection in women is debatable [34]. While a role for condom use in reducing HPV infection in FSWs has been reported $[11,27,30]$, there is an argument that condoms may not completely cover the infected areas, thus rendering insufficient protection against HPV infection [19]. In this study, no significant difference was found in HPV prevalence between condom users and non-condom users, supporting a limited capacity of condoms in HPV protection.

In this study, HPV infection in FSWs in Shenyang was associated with all other socio-demographic, medical and behavioral variables including marital status, number of abortions, sexual disease history, use of contraception pills and other medications, age of puberty, and previous gynecologic and obstetric examinations. Nevertheless, these findings need to be further validated in studies with a large sample size.

This study has several limitations. First, ThinPap test and cytological evaluation were performed only once on both FSWs and control subjects. This cannot totally rule out the possibility of miss the true abnormalities. Second, in evaluating the usefulness of condom use in protecting against HPV infection, only the information on 'current condom use' at the time of interview was collected. This might not represent a complete picture. A more accurate answer would have been obtained if condom use had been stratified into three scenarios: no condom use either in the past or at the present; condom use always; and condom use sometimes.

\section{Conclusions}

HPV prevalence and cervical lesion incidence were substantially higher in FSWs than in healthy control subjects in Shenyang. Falling in the upper range of the reported values across different countries, HPV prevalence detected in FSWs in this study was similar to that for FSWs in southeast Huzhou but higher than that for FSWs in southwest Guangxi within China. Age of first sexual intercourse and menopause were two independent risk factors for HPV infection in our sample of FSWs. Public health endeavor is urgently needed to reduce the risk of HPV infection in FSWs in the northeast Chinese city of Shenyang. 


\section{Competing interests}

None of the authors has any potential financial conflict of interest related to this work.

\section{Authors' contributions}

HJ conceptualized the study, designed the questionnaire survey, performed HPV testing and prepared the manuscript. XW and ZL participated in HPV testing in healthy control subjects, data analysis and discussion on manuscript preparation. LL did oversee the execution of the study and manuscript preparation. All authors read and approved the final manuscript.

\section{Acknowledgements}

We would like to thank Dr. Ziming Yu for assistance with the manuscript preparation.

Received: 10 March 2015 Accepted: 16 July 2015

Published online: 23 July 2015

\section{References}

1. WHO: Human papillomavirus (HPV) and cervical cancer. http://www.who.int/ mediacentre/factsheets/fs380/en/.

2. Davey DD. Cervical cytology classification and the Bethesda System. Cancer J. 2003;9(5):327-34

3. Bosch FX, Manos MM, Munoz N, Sherman M, Jansen AM, Peto J, et al. Prevalence of human papillomavirus in cervical cancer: a worldwide perspective. International biological study on cervical cancer (IBSCC) Study Group. J Natl Cancer Inst. 1995;87(11):796-802.

4. Bennett C, Kuhn AE, Haverkos HW. Human papillomavirus and tar hypothesis for squamous cell cervical cancer. J Biosci. 2010;35(3):331-7.

5. Sellors JW, Karwalajtys TL, Kaczorowski J, Mahony JB, Lytwyn A, Chong S, et al. Survey of HPViOWG: Incidence, clearance and predictors of human papillomavirus infection in women. CMAJ. 2003;168(4):421-5.

6. Winer RL, Lee SK, Hughes JP, Adam DE, Kiviat NB, Koutsky LA. Genital human papillomavirus infection: incidence and risk factors in a cohort of female university students. Am J Epidemiol. 2003;157(3):218-26.

7. Soohoo M, Blas M, Byraiah G, Carcamo C, Brown B. Cervical HPV infection in female sex workers: a global perspective. Open AIDS J. 2013;7:58-66.

8. Li HM, Liang GJ, Yin YP, Wang QQ, Zheng ZJ, Zhou JJ, et al. Prevalence and genotype distribution of human papillomavirus infection among female sex workers in Guangxi, China: implications for interventions. J Med Virol. 2012;84(5):798-803.

9. Shikova E, Todorova I, Ganchev G, Kouseva-Dragneva V, Kalascheva-Zaimova P. Prevalence of human papillomavirus infection among female sex workers in Bulgaria. Int J STD AIDS. 2011;22(5):278-80.

10. del Amo J, Gonzalez C, Belda J, Fernandez E, Martinez R, Gomez I, et al. Prevalence and risk factors of high-risk human papillomavirus in female sex workers in Spain: differences by geographical origin. J Women's Health. 2009;18(12):2057-64.

11. Wang X, Gu D, Lou B, Xu B, Qian F, Chen Y. Hospital-based prevalence of high-risk cervical HPV types infecting the general population and female sex workers in Huzhou, China. Int J Gynaecol Obstet. 2013;120(1):37-41.

12. Duggan MA, Khalil M, Brasher PM, Nation JG. Comparative study of the ThinPrep Pap test and conventional cytology results in a Canadian cohort. Cytopathology. 2006;17(2):73-81.

13. Lynge E, Rebolj M. Primary HPV screening for cervical cancer prevention: results from European trials. Nat Rev Clin Oncol. 2009;6(12):699-706.

14. Eide ML, Debaque H. HPV detection methods and genotyping techniques in screening for cervical cancer. Ann Pathol. 2012;32(6):e15-23. 401-409.

15. de Cremoux P, Coste J, Sastre-Garau X, Thioux M, Bouillac C, Labbe S, et al. Efficiency of the hybrid capture 2 HPV DNA test in cervical cancer screening. A study by the French Society of Clinical Cytology. Am J Clin Pathol. 2003;120(4):492-9.

16. Poljak M, Ostrbenk A, Seme K, Ucakar V, Hillemanns P, Bokal EV, et al. Comparison of clinical and analytical performance of the Abbott Realtime High Risk HPV test to the performance of hybrid capture 2 in populationbased cervical cancer screening. J Clin Microbiol. 2011;49(5):1721-9.

17. Berman NR. Cervical cancer screening today. The role of HPV DNA testing. Adv Nurse Pract. 2006;14(4):24-9. quiz 30.

18. Hernandez BY, Vu Nguyen T. Cervical human papillomavirus infection among female sex workers in southern Vietnam. Infect Agents Cancer. 2008;3:7.
19. Marek E, Dergez T, D'Cruz G, Bozsa S, Cseh A, Szilard I, et al. Human papillomavirus infections among Hungarian female sex workers. Eur J Cancer Care (Engl). 2014;23(1):65-75.

20. Brown B, Blas MM, Cabral A, Byraiah G, Guerra-Giraldez C, Sarabia-Vega V, et al. Human papillomavirus prevalence, cervical abnormalities and risk factors among female sex workers in Lima, Peru. Int J STD AIDS. 2012;23(4):242-7.

21. Miyashita M, Agdamag DM, Sasagawa T, Matsushita K, Salud LM, Salud CO, et al. High-risk HPV types in lesions of the uterine cervix of female commercial sex workers in the Philippines. J Med Virol. 2009;81(3):545-51.

22. Hoang HT, Ishizaki A, Nguyen CH, Tran VT, Matsushita K, Saikawa K, et al. Infection with high-risk HPV types among female sex workers in northern Vietnam. J Med Virol. 2013;85(2):288-94.

23. Choi BS, Kim O, Park MS, Kim KS, Jeong JK, Lee JS. Genital human papillomavirus genotyping by HPV oligonucleotide microarray in Korean commercial sex workers. J Med Virol. 2003;71(3):440-5.

24. Tideman RL, Thompson C, Rose B, Gilmour S, Marks C, van Beek I, et al. Cervical human papillomavirus infections in commercial sex workers-risk factors and behaviours. Int J STD AIDS. 2003;14(12):840-7.

25. Chandeying V, Garland SM, Tabrizi SN. Prevalence and typing of human papilloma virus (HPV) among female sex workers and outpatient women in southern Thailand. Sex Health. 2006;3(1):11-4.

26. Chan R, Khoo L, Ho TH, Koh CF, Lee IW, Yam KL, et al. A comparative study of cervical cytology, colposcopy and PCR for HPV in female sex workers in Singapore. Int J STD AIDS. 2001;12(3):159-63.

27. Smith JS, Van Damme K, Randrianjafisamindrakotroka N, Ting J, Rabozakandraina T, Randrianasolo BS, et al. Human papillomavirus and cervical neoplasia among female sex workers in Madagascar. Int J Gynecol Cancer. 2010;20(9):1593-6.

28. Wong WC, Wun YT, Chan KW, Liu Y. Silent killer of the night: a feasibility study of an outreach well-women clinic for cervical cancer screening in female sex workers in Hong Kong. Int J Gynecol Cancer. 2008;18(1):110-5.

29. Leung KM, Yeoh GP, Cheung HN, Fong FY, Chan KW. Prevalence of abnormal Papanicolaou smears in female sex workers in Hong Kong. Hong Kong Med J. 2013;19(3):203-6.

30. Kjaer SK, Svare El, Worm AM, Walboomers JM, Meijer CJ, van den Brule AJ. Human papillomavirus infection in Danish female sex workers. Decreasing prevalence with age despite continuously high sexual activity. Sex Transm Dis. 2000;27(8):438-45.

31. Sivro A, Lajoie J, Kimani J, Jaoko W, Plummer FA, Fowke K, et al. Age and menopause affect the expression of specific cytokines/chemokines in plasma and cervical lavage samples from female sex workers in Nairobi, Kenya. Immun Ageing. 2013;10(1):42.

32. Kaderli $\mathrm{R}$, Schnuriger $\mathrm{B}$, Brugger LE. The impact of smoking on HPV infection and the development of anogenital warts. Int J Color Dis. 2014;29(8):899-908.

33. Nunez JT, Delgado M, Pino G, Giron H, Bolet B. Smoking as a risk factor for preinvasive and invasive cervical lesions in female sex workers in Venezuela. Int J Gynaecol Obstet. 2002;79(1):57-60.

34. Chelimo C, Wouldes TA, Cameron LD, Elwood JM. Risk factors for and prevention of human papillomaviruses (HPV), genital warts and cervical cancer. J Infect. 2013;66(3):207-17.

\section{Submit your next manuscript to BioMed Central and take full advantage of:}

- Convenient online submission

- Thorough peer review

- No space constraints or color figure charges

- Immediate publication on acceptance

- Inclusion in PubMed, CAS, Scopus and Google Scholar

- Research which is freely available for redistribution 